\title{
Smart Loads for Voltage Control in Distribution Networks
}

\author{
Zohaib Akhtar, Student Member, IEEE, Balarko Chaudhuri, Senior Member, IEEE, \\ and Shu Yuen Ron Hui, Fellow, IEEE
}

\begin{abstract}
This paper shows that the smart loads (SLs) could be effective in mitigating voltage problems caused by photovoltaic (PV) generation and electric vehicle $(\mathrm{EV})$ charging in low-voltage (LV) distribution networks. Limitations of the previously reported SL configuration with only series reactive compensator (SLQ) (one converter) is highlighted in this paper. To overcome these limitations, an additional shunt converter is used in back-to-back (B2B) configuration to support the active power exchanged by the series converter, which increases the flexibility of the SL without requiring any energy storage. Simulation results on a typical U.K. LV distribution network are presented to compare the effectiveness of an SL with B2B converters (SLBCs) against an SLQ in tackling under- and over-voltage problems caused by $E V$ or PV. It is shown that SLBCs can regulate the main voltage more effectively than SLQs especially under overvoltage condition. Although two converters are required for each SLBC, it is shown that the apparent power capacity of each converter is required to be significantly less than that of an equivalent SLQ.
\end{abstract}

Index Terms-Demand response, distribution network, electric spring (ES), electric vehicles (EVs), photovoltaic (PV), smart load (SL), voltage control.

\section{INTRODUCTION}

$\mathbf{I}$ NCREASING use of distributed generations (DGs) such as roof-top photovoltaic (PV) generation would cause over-voltage problem in low-voltage and/or medium voltage (LV/MV) distribution networks [1]. On the other hand, charging the growing fleet of electric vehicles (EVs) during the night could lead to under voltage problem even during otherwise offpeak hours [2]. Such voltage problems could potentially become unacceptable with increasing penetration of $\mathrm{PVs} / \mathrm{EVs}$.

There are a number of ways in which an acceptable voltage profile could be maintained along the LV/MV feeders.

Manuscript received February 25, 2015; revised August 4, 2015; accepted September 24, 2015. Date of publication October 26, 2015; date of current version February 16, 2017. This work was supported in part by the Commonwealth Scholarship Commission, Engineering and Physical Science Research Council, U.K., under Grant EP/K036327/1, and in part by the Hong Kong Research Grant Council under the Theme-based project T23-701/14-N. Paper no. TSG-00232-2015.

Z. Akhtar and B. Chaudhuri are with the Department of Electrical and Electronic Engineering, Imperial College London, London SW7 2AZ, U.K. (e-mail: z.akhtar13@imperial.ac.uk; b.chaudhuri@imperial.ac.uk).

S. Y. R. Hui is with the Department of Electrical and Electronic Engineering, Imperial College London, London SW7 2AZ, U.K., and also with the Department of Electrical and Electronic Engineering, University of Hong Kong, Hong Kong (e-mail: r.hui@ imperial.ac.uk).

Color versions of one or more of the figures in this paper are available online at http://ieeexplore.ieee.org.

Digital Object Identifier 10.1109/TSG.2015.2486139
This includes but is not limited to optimal dispatch control of DGs [3] (not applicable for nondispatchable sources such as PV), transformer tap changer control, load control [4], control of the PV/EV inverters [5], matching the EV charging periods to peak PV outputs [6], the use of distributed energy storage [7], [8], etc. Active network management could also play a part in voltage control [9]. These options are exercised over different time scales and proper coordination should be ensured wherever appropriate [5].

Voltages in the LV network depend on both active and reactive power flows due to relatively high values of system $R / X$ ratio in the LV networks. Hence, reactive shunt compensators alone are not very effective in controlling the voltage at the LV network level unlike the high voltage system. Control over active power exchange in addition to reactive power is required which is possible through either energy storage and/or control of active power consumption of the loads. While the former could be quite expensive, the latter could be effectively used with certain types of loads (e.g., electric heating, refrigerator, passive LED lighting systems, appliances with motors such as oven, washing machine, and dish washer) without noticeable impact. Control of loads for demand response is usually discontinuous (on/off control) and is exercised for peak shaving, load following, etc. An alternative approach is to employ continuous control over the power consumption of a load by decoupling it from the supply mains using a series compensator (converter) in between [10]. In LV/MV networks, a reactive series compensator (also referred to as "electric spring (ES)" in [10]) connected between the supply mains and a voltage-dependent load has been shown to be more effective in controlling the voltage than an equivalent shunt compensator [11], [12]. A series compensator allows the voltage at the terminal of the load and hence, its active power consumption to be controlled over a wider range (within the allowed limit) to regulate the mains voltage.

The voltage regulation capability of an smart load (SL) with only reactive compensation (SLQ) is limited by the fact that its active and reactive power consumption are dependent on each other (as discussed later in Section II). Independent control over both active and reactive power consumption of an SL is only possible if both the magnitude and the phase angle of the voltage injected by the compensator is controlled unlike an SLQ where only the magnitude of the compensator voltage can be controlled with the phase angle (with respect to current) maintained at $\pm 90^{\circ}$. Relaxing this quadrature constraint on phase angle would result in both active and reactive 
power exchange with the compensator which is termed as an SL with active and reactive compensation (SLPQ). One way to support the active power exchanged is to use some form of energy storage (e.g., batteries and super-capacitors) in the compensator which is henceforth referred to as an SL with energy storage (SLES). Alternatively, an additional shunt converter could be used in back-to-back (B2B) configuration so that the supply/mains can support the active power exchanged by the compensator (converter) connected in series with the load [13]. This is referred to as an SL with B2B converter (SLBC) throughout the rest of this paper. An SLBC not only obviates the need for energy storage (which is expensive) at the expense of an additional converter, but also has larger capability compared to an SLES as shown later in this paper.

The effectiveness of SLBCs is demonstrated in this paper by comparing its voltage regulation performance against SLQs. The active and reactive power $(P-Q)$ capability and the control philosophy for an SLBC are discussed for the first time. A case study on a segment of a typical LV distribution network from the Department of Trade and Industry, U.K. [14], is presented. PV panels and EV charging facility are installed at each load point in such a way that the peak power output of the PV panels causes over-voltage problems during the day time while the charging of EVs leads to under-voltage situation during the night. These extreme cases are considered to illustrate the benefits of SLBCs over SLQs which should facilitate the integration of DGs and EVs. The role of SLQs in voltage regulation has been presented previously [10], [11], [15]. In this paper, the limitations of SLQs in LV networks (with high $R / X$ ratio) are highlighted and SLBCs are presented as an effective alternative.

The converter capacity for an SLQ is compared against that of an SLBC in order to achieve similar performance. Although two converters are required for each SLBC, it is shown that the apparent power capacity of each converter is required to be considerably less than that of an equivalent SLQ. Alongside the nominal case, voltage regulation performance with SLQs and SLBCs are compared for different $R / X$ ratios and load power factors (pfs) to substantiate the claims in this paper.

\section{SMART LOAD}

Loads can be broadly classified into critical loads which require a tightly regulated supply voltage, and noncritical (NC) ones which can tolerate a relatively large voltage variation with little or no noticeable impact on the consumers. Some of these NC loads draw a constant power over a wide range of supply voltage variation (e.g., air-conditioners), while for others, the power consumption is dependant on the terminal voltage. This includes heaters [16], lighting (especially, passive LED lighting systems [17]), and small motors with no stalling problems (e.g., fans, ovens, dish washers, and dryers) [18], where it is possible, in principle, to exercise a continuous variation in active power consumed by controlling the terminal voltage [15]. Such a voltage-dependant NC load can be converted into an SL by inserting a voltage compensator (or ES) in series between the supply/mains and the load itself [10].

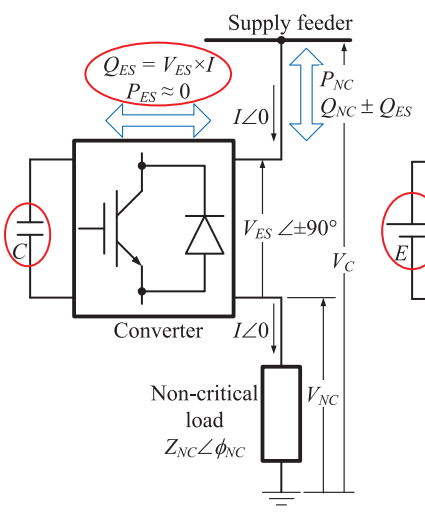

(a)

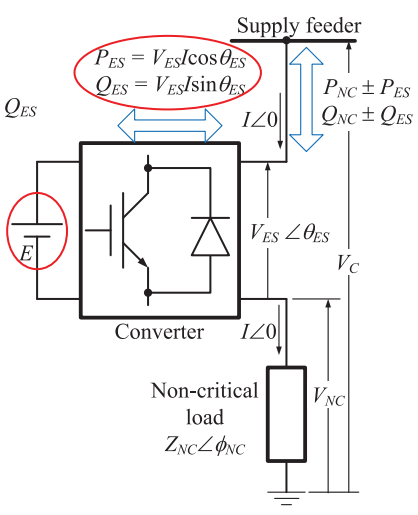

(b)
Fig. 1. (a) SLQ. (b) SLES.

Depending on the type of compensator used, an SL can be classified as follows.

\section{A. Smart Load With Only Reactive Compensation}

A compensator or ES is a converter with a dc link that injects a voltage with controllable magnitude $\left(V_{\mathrm{ES}}\right)$ and phase angle $\left(\theta_{\mathrm{ES}}\right)$ in series with the $\mathrm{NC}$ load as shown in Fig. 1(a). The voltage $\left(V_{\mathrm{NC}}\right)$ across the $\mathrm{NC}$ loads is thus controlled (within allowable bounds) and the active/reactive power consumed by it is modulated. If the injected voltage is maintained in quadrature with the current flow $\left(\theta_{\mathrm{ES}}= \pm 90^{\circ}\right)$, there is no active power contribution from the compensator. This type of SL is called SLQ [10], [11], [15] and is shown in Fig. 1(a).

Neglecting the power loss in the converter, the active power consumption of the SL $\left(P_{\mathrm{SL}}\right)$ is equal to the active power consumption of the NC load $\left(P_{\mathrm{NC}}\right)$. The total reactive power of the $\mathrm{SL}\left(Q_{\mathrm{SL}}\right)$ is equal to the algebraic sum of reactive powers of the compensator $\left(Q_{\mathrm{ES}}\right)$ and the $\mathrm{NC}$ load $\left(Q_{\mathrm{NC}}\right)$. The converter is controlled to maintain $V_{\mathrm{ES}}$ and $\theta_{\mathrm{ES}}$, where only the magnitude of the inserted voltage $\left(V_{\mathrm{ES}}\right)$ is varied while keeping the phase angle $\left(\theta_{\mathrm{ES}}\right)$ at $\pm 90^{\circ}$. Simultaneous control of both active and reactive power of SL is not possible with an SLQ. Either the active or the reactive power of the SL can be controlled (depending on the $R / X$ ratio of the system) to control the supply voltage. An SLQ can also contribute to primary frequency by changing its active power consumption but that will result in variation in the supply voltage [12].

\section{B. Smart Load With Active and Reactive Compensation}

For an SLQ, the phase angle $\theta_{\mathrm{ES}}$ of the voltage injected by the compensator is maintained at $\pm 90^{\circ}$ which restricts its capability (as shown later in Section II-C). If the phase angle $\theta_{\mathrm{ES}}$ is allowed to vary freely, the compensator would exchange active power as well resulting in an SLPQ. For an SLPQ, both the magnitude and phase angle of the voltage injected by the compensator can be controlled independently. This enables effective voltage control irrespective of the $R / X$ ratio of the system and load power factor (pf) (as shown later in Section II-C). An SLPQ can also be used for simultaneous control of both voltage and frequency by modulating its active and reactive power consumption, which is not possible 
with an SLQ. However, these benefits of an SLPQ come at the expense of active power exchange with the compensator which can be supported from either an energy storage (e.g., battery or super capacitor) or the supply itself through a B2B converter arrangement as described next.

1) Smart Load With Energy Storage: An SLES is shown in Fig. 1(b). The total active (or reactive) power consumption of the SL $P_{\mathrm{SL}}\left(Q_{\mathrm{SL}}\right)$ is equal to the algebraic sum of the active (reactive) power of the compensator $P_{\mathrm{ES}}\left(Q_{\mathrm{ES}}\right)$ and the $\mathrm{NC}$ load $P_{\mathrm{NC}}\left(Q_{\mathrm{NC}}\right)$. Independent control of the active and the reactive power is possible by controlling the magnitude $V_{\mathrm{ES}}$ and the phase angle $\theta_{\mathrm{ES}}$ of the inserted voltage. In addition, auxiliary control of the state-of-charge (SOC) of the energy storage is required.

The critical consideration toward realizing an SLES is the rating of the energy storage in terms of both high power (for fast transient voltage control) and high energy (for sustained under- or over-voltage situations). Depending on the type of the disturbance (under- or over-voltage condition), the energy storage would have to be prepared to either charge or discharge to cause a resultant decrease or increase in active power consumption of the SL. Thus, under normal conditions, the SOC should be maintained half way between the upper and the lower SOC limits of the storage device in order to allow equal margin in both directions. This results in doubling of the energy storage rating.

Following an event, the energy storage in all the compensators must be restored to its nominal SOC. Simultaneous restoration for all SLs would trigger additional voltage (and possibly frequency) disturbances in the system. For these reasons, an SLES is a viable option only for primary frequency control support where the time frame is tens of seconds. However, it is virtually ruled out for voltage control as the potentially longer time durations would require prohibitively large energy storage ratings. Moreover, the active and reactive power capability of an SLES could be quite limited as discussed later in Section II-C which reduces their effectiveness.

2) Smart Load With Back-to-Back Converters: In an SLBC, the energy storage is replaced by a bidirectional ac-to-dc converter with the ac side connected to the power grid. The configuration is shown in Fig. 2 and is somewhat similar to an unified power quality conditioner which uses shunt-series converters [19], except that one converter (i.e., converter 1) is connected in series with the NC load and an input control is adopted in the converter. This converter is set to control the magnitude $V_{\mathrm{ES}}$ and the phase angle $\theta_{\mathrm{ES}}$ of the inserted voltage. The other converter (i.e., converter 2) connected in parallel/shunt to the supply maintains the voltage $V_{\mathrm{dc}}$ across the dc link and thereby, supports the active power exchanged by the series converter (converter 1).

The parallel converter can be operated at unity pf (i.e., only active power exchange) to minimize its apparent power rating. Ideally, the active power supplied (or consumed) by the series compensator $\left(P_{\mathrm{ES}}\right)$ will be the same as the power consumed (supplied) by the shunt converter from the supply. Hence, the total active power consumption of the $\mathrm{SL}\left(P_{\mathrm{SL}}\right)$ will be the same as that of the NC load $\left(P_{\mathrm{NC}}\right)$.

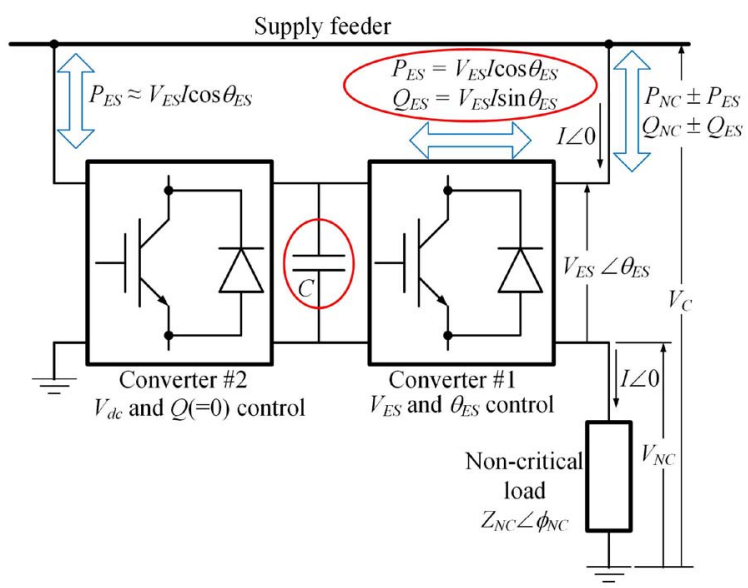

Fig. 2. SLBC.

As no energy storage is required in SLBC, it can support under- or over-voltage events of longer duration depending on the nature of the NC loads. It also provides more flexibility in terms of control over active and reactive power consumption of the SL as explained next.

\section{Active and Reactive Power Capability}

It is important to estimate the active and reactive power capabilities of the SLs in order to evaluate their effectiveness in voltage (and primary frequency) control [12]. From Figs. 1 and 2, the voltage across the supply voltage $\left(V_{C}\right)$ can be expressed as the phasor sum of the NC load voltage $\left(V_{\mathrm{NC}}\right)$ and the compensator voltage $\left(V_{\mathrm{ES}}\right)$

$$
V_{C} \angle \theta_{C}=V_{\mathrm{NC}} \angle \phi_{\mathrm{NC}}+V_{\mathrm{ES}} \angle \theta_{\mathrm{ES}}
$$

where $\theta_{C}$ is the phase angle of the supply voltage, $\theta_{\mathrm{ES}}$ is the phase angle of the compensator voltage, and $\phi_{\mathrm{NC}}$ is the NC load impedance angle. The above equation can be expressed as

$$
\begin{aligned}
V_{C}^{2}= & V_{\mathrm{NC}}^{2}+V_{\mathrm{ES}}^{2}+2 V_{\mathrm{NC}} V_{\mathrm{ES}} \cos \left(\phi_{\mathrm{NC}}-\theta_{\mathrm{ES}}\right) \\
V_{\mathrm{NC}}= & -V_{\mathrm{ES}} \cos \left(\phi_{\mathrm{NC}}-\theta_{\mathrm{ES}}\right) \\
& \pm \sqrt{V_{C}^{2}-V_{\mathrm{ES}}^{2} \sin \left(\phi_{\mathrm{NC}}-\theta_{\mathrm{ES}}\right)^{2}} \\
= & F\left(V_{C}, V_{\mathrm{ES}}, \theta_{\mathrm{ES}}\right) .
\end{aligned}
$$

If $V_{C}$ is assumed to be 1 p.u., the value(s) of $V_{\mathrm{NC}}$ corresponding to any given values of $V_{\mathrm{ES}}$ and $\theta_{\mathrm{ES}}$ can be calculated using (3). Only real and positive values of $V_{\mathrm{NC}}$ are considered and used to calculate the active power $\left(P_{\mathrm{SL}}\right)$, the reactive power $\left(Q_{\mathrm{SL}}\right)$, the change in active power $\left(\Delta P_{\mathrm{SL}}\right)$, and the change in reactive power $\left(\Delta Q_{\mathrm{SL}}\right)$ of the SL for different values of $V_{\mathrm{ES}}$ for all possible phase angles $\left(\theta_{\mathrm{ES}}\right)$. The corresponding values of the active $\left(P_{\mathrm{ES}}\right)$ and the reactive $\left(Q_{\mathrm{ES}}\right)$ compensation required can also be determined subsequently.

The capabilities of three types of SLs (i.e., SLQ, SLES, and $\mathrm{SLBC})$ rated at 1.0 p.u. $\left(Z_{\mathrm{NC}}=1\right.$ p.u.) are compared under two different pfs of the NC load assuming 1.0 p.u. supply voltage ( $V_{C}=1$ p.u. $)$. 


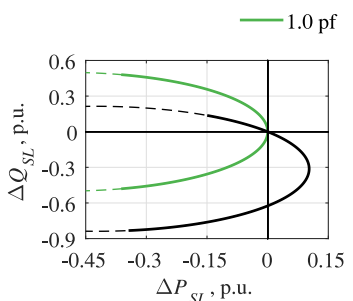

(a)

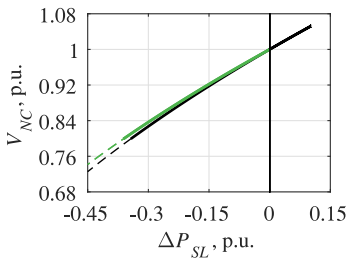

(c)

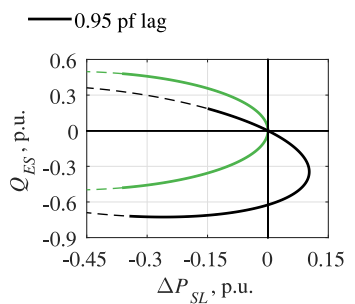

(b)

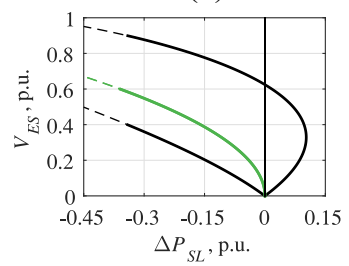

(d)
Fig. 3. Capability of SLQ: change in (a) reactive power consumption of SL (y-axis), (b) reactive compensation required (y-axis), (c) NC load voltage ( $y$-axis), and (d) compensation (ES) voltage ( $y$-axis) for change in active power consumption of SL ( $x$-axis) for two different pfs.

1) SLQ Capability: In order to draw the capability curve for SLQ, all possible values of $V_{\mathrm{NC}}$ are calculated from (3) over a range of values of $V_{\mathrm{ES}}(0-1$ p.u.) under both possible phase angles $\left(\theta_{\mathrm{ES}}=+90^{\circ}\right.$ and $\left.\theta_{\mathrm{ES}}=-90^{\circ}\right)$. This can be used to find out the values of the active and the reactive powers of SLQ as given by the following equations:

$$
\begin{aligned}
P_{\mathrm{SL}}=P_{\mathrm{NC}} & =\frac{V_{\mathrm{NC}}^{2} \cos \left(\phi_{\mathrm{NC}}\right)}{Z_{\mathrm{NC}}} \\
Q_{\mathrm{SL}} & =Q_{\mathrm{ES}}+Q_{\mathrm{NC}} \\
& =\frac{ \pm V_{\mathrm{ES}} V_{\mathrm{NC}}}{Z_{\mathrm{NC}}}+\frac{V_{\mathrm{NC}}^{2} \sin \left(\phi_{\mathrm{NC}}\right)}{Z_{\mathrm{NC}}}
\end{aligned}
$$

where the positive and negative sign of $Q_{\mathrm{ES}}$ corresponds to the inductive and capacitive compensation modes, respectively. If $P_{\text {SL0 }}$ and $Q_{\text {SL0 }}$ are the values of SL active and reactive powers with no compensation $\left(V_{\mathrm{ES}}=0\right)$, one can write

$$
\begin{aligned}
\Delta P_{\mathrm{SL}} & =P_{\mathrm{SL}}-P_{\mathrm{SL} 0} \\
& =\frac{V_{\mathrm{NC}}^{2} \cos \left(\phi_{\mathrm{NC}}\right)}{Z_{\mathrm{NC}}}-\frac{V_{C}^{2} \cos \left(\phi_{\mathrm{NC}}\right)}{Z_{\mathrm{NC}}} \\
\Delta Q_{\mathrm{SL}} & =Q_{\mathrm{SL}}-Q_{\mathrm{SL} 0} \\
& =\frac{ \pm V_{\mathrm{ES}} V_{\mathrm{NC}}}{Z_{\mathrm{NC}}}+\frac{V_{\mathrm{NC}}^{2} \sin \left(\phi_{\mathrm{NC}}\right)}{Z_{\mathrm{NC}}}-\frac{V_{C}^{2} \sin \left(\phi_{\mathrm{NC}}\right)}{Z_{\mathrm{NC}}} .
\end{aligned}
$$

The capability of an SLQ is shown in Fig. 3 for two different pfs of the NC load. The thin dotted lines represent the whole range without any constraint on the magnitude of $V_{\mathrm{NC}}$, while the thick solid lines represent the region in which $V_{\mathrm{NC}}$ is limited within the range of $0.8-1.2$ p.u. There is no restriction on the ES reactive power $\left(Q_{\mathrm{ES}}\right)$.

The change in the active $\left(\Delta P_{\mathrm{SL}}\right)$ and reactive $\left(\Delta Q_{\mathrm{SL}}\right)$ powers are shown in Fig. 3(a). For unity pf NC load (green trace), there are no positive values for $\Delta P_{\mathrm{SL}}$ as $V_{\mathrm{NC}}$ cannot be larger than the supply voltage [Fig. 3(c)] for any value of the $V_{\mathrm{ES}}$ and $\theta_{\mathrm{ES}}= \pm 90^{\circ}$. Hence, a unity pf NC load can only be used to tackle under-voltage conditions which requires a reduction in active power consumption (i.e., negative $\Delta P_{\mathrm{SL}}$ ) of the SL.
For a 0.95 lagging power [shown in Fig. 3(a) (black trace)], the active power consumption of the SL can be varied in both directions to tackle under- or over-voltage events. Out of the two possible values of $V_{\mathrm{ES}}$ which yields the same $\Delta P_{\mathrm{SL}}$ [Fig. 3(d)], the smaller value should be used to ensure the minimum rating of the compensator. The capability curve for 0.95 lagging pf in Fig. 3(a) is not symmetrical about the horizontal axis. There is a smaller operating range on the inductive compensation side if the SLQ is set to operate in the reactive power control mode.

The maximum positive value of $\Delta P_{\mathrm{SL}}$ occurs at the point when the inductive reactive power consumption of the NC load is equal to the capacitive reactive power of the converter. The current flowing through the SLQ is maximum at this point and is chosen as the capacity of the SLQ converter for the case study in Section III.

One limitation of an SLQ is either the active or reactive power consumption of the SL can be controlled. It can be seen from Fig. 3(b) that $Q_{\mathrm{ES}}$ is mostly negative for positive values of $\Delta P_{\mathrm{SL}}$. An increase in active power consumption in this zone results in a decrease in reactive power of the SL [Fig. 3(a)]. This could be a problem in the case of an overvoltage event in LV/MV networks with a moderate $R / X$ ratio where both active and reactive power exchanges are equally critical for voltage control.

2) SLES Capability: After calculating the values of $V_{\mathrm{NC}}$ corresponding to all possible values of $V_{\mathrm{ES}}$ and $\theta_{\mathrm{ES}}$, one can use (12)-(19) to find out $\Delta P_{\mathrm{SL}}$ and $\Delta Q_{\mathrm{SL}}$ for an SLES

$$
\begin{aligned}
P_{\mathrm{SL}}= & P_{\mathrm{ES}}+P_{\mathrm{NC}} \\
= & \frac{V_{\mathrm{ES}} V_{\mathrm{NC}} \cos \left(\theta_{\mathrm{ES}}\right)}{Z_{\mathrm{NC}}}+\frac{V_{\mathrm{NC}}^{2} \cos \left(\phi_{\mathrm{NC}}\right)}{Z_{\mathrm{NC}}} \\
Q_{\mathrm{SL}}= & Q_{\mathrm{ES}}+Q_{\mathrm{NC}} \\
= & \frac{V_{\mathrm{ES}} V_{\mathrm{NC}} \sin \left(\theta_{\mathrm{ES}}\right)}{Z_{\mathrm{NC}}}+\frac{V_{\mathrm{NC}}^{2} \sin \left(\phi_{\mathrm{NC}}\right)}{Z_{\mathrm{NC}}} \\
\Delta P_{\mathrm{SL}}= & P_{\mathrm{SL}}-P_{\mathrm{SL} 0} \\
= & \frac{V_{\mathrm{ES}} V_{\mathrm{NC}} \cos \left(\theta_{\mathrm{ES}}\right)}{Z_{\mathrm{NC}}}+\frac{V_{\mathrm{NC}}^{2} \cos \left(\phi_{\mathrm{NC}}\right)}{Z_{\mathrm{NC}}} \\
& -\frac{V_{C}^{2} \cos \left(\phi_{\mathrm{NC}}\right)}{Z_{\mathrm{NC}}} \\
Q_{\mathrm{SL}}= & Q_{\mathrm{SL}}-Q_{\mathrm{SL} 0} \\
= & \frac{V_{\mathrm{ES}} V_{\mathrm{NC}} \sin \left(\theta_{\mathrm{ES}}\right)}{Z_{\mathrm{NC}}}+\frac{V_{\mathrm{NC}}^{2} \sin \left(\phi_{\mathrm{NC}}\right)}{Z_{\mathrm{NC}}} \\
& -\frac{V_{C}^{2} \sin \left(\phi_{\mathrm{NC}}\right)}{Z_{\mathrm{NC}}} .
\end{aligned}
$$

The capability curves for an SLES are shown in Fig. 4. The subplots in the left column are for a unity pf load, while the subplots in the right column are for a 0.95 lagging pf load. The range of these capability curves is limited by the apparent power capacity $S_{\mathrm{ES}}$ of the compensator which is considered to be 0.2 p.u. Also, the variation in voltage across the NC loads is limited to $\pm 20 \%$ as in the case of SLQ.

The capability curves for an SLES cover all four quadrants allowing any combination of change in its active and reactive power consumption unlike an SLQ. The capability is constrained for negative $\Delta P_{\mathrm{SL}}$ due to the lower limit of the 


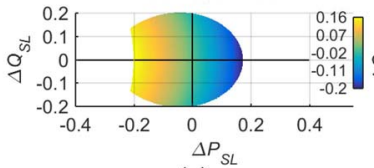

(a)

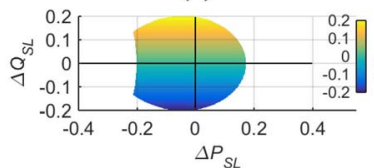

(c)

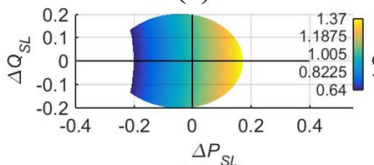

(e)

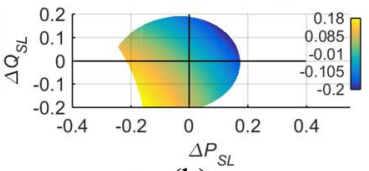

(b)

(d)

(f)
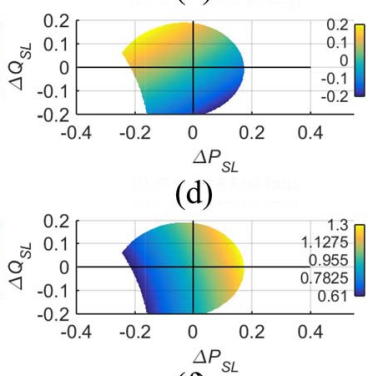

Fig. 4. Capability of SLES. (a) and (b) Active compensation required. (c) and (d) Reactive compensation required. (e) and (f) Active power consumption of NC load corresponding to the change in active power ( $x$-axis) and reactive power ( $y$-axis) of an SLES for two different pfs.

$\mathrm{NC}$ load voltage $\left(V_{\mathrm{NC}}>0.8\right.$ p.u.). However, similar constraint due to the upper limit of the $\mathrm{NC}$ load voltage $\left(V_{\mathrm{NC}}<1.2\right.$ p.u. $)$ is not evident as the converter capacity is the dominant factor for positive $\Delta P_{\mathrm{SL}}$. The active $\left(P_{\mathrm{ES}}\right)$ and reactive $\left(Q_{\mathrm{ES}}\right)$ powers required by the converter (ES) for operation in different quadrants (for both pfs) are shown in Fig. 4(a)-(d).

For unity pf load, Fig. 4(a) shows that the active power exchanged by the compensator $\left(P_{\mathrm{ES}}\right)$ has an opposite sign to the change active power consumption of the $\mathrm{NC}$ load $\left(\Delta P_{\mathrm{NC}}\right)$ which is proportional to $V_{\mathrm{NC}}$ as shown in Fig. 4(e). For 0.95 lagging pf load, this is also true for most regions as shown in Fig. 4(b) and (f). As $\Delta P_{\mathrm{SL}}$ is equal to the algebraic sum of $P_{\mathrm{ES}}$ and $\Delta P_{\mathrm{NC}}$, it can be seen that a larger value of $\Delta P_{\mathrm{NC}}$ could result in an overall smaller value of $\Delta P_{\mathrm{SL}}$. This limitation of an SLES can be overcome by an SLBC where $\Delta P_{\mathrm{SL}}$ is equal to $\triangle P_{\mathrm{NC}}$ as described next.

3) SLBC Capability: The values of $\Delta P_{\mathrm{SL}}$ and $\Delta Q_{\mathrm{SL}}$ for an SLBC can be calculated as follows:

$$
\begin{aligned}
P_{\mathrm{SL}}= & P_{\mathrm{NC}}=\frac{V_{\mathrm{NC}}^{2} \cos \left(\phi_{\mathrm{NC}}\right)}{Z_{\mathrm{NC}}} \\
Q_{\mathrm{SL}}= & Q_{\mathrm{ES}}+Q_{\mathrm{NC}} \\
= & \frac{V_{\mathrm{ES}} V_{\mathrm{NC}} \sin \left(\theta_{\mathrm{ES}}\right)}{Z_{\mathrm{NC}}}+\frac{V_{\mathrm{NC}}^{2} \sin \left(\phi_{\mathrm{NC}}\right)}{Z_{\mathrm{NC}}} \\
\Delta P_{\mathrm{SL}}= & P_{\mathrm{SL}}-P_{\mathrm{SL} 0}^{2} \\
= & \frac{V_{\mathrm{NC}}^{2} \cos \left(\phi_{\mathrm{NC}}\right)}{Z_{\mathrm{NC}}}-\frac{V_{C}^{2} \cos \left(\phi_{\mathrm{NC}}\right)}{Z_{\mathrm{NC}}} \\
\Delta Q_{\mathrm{SL}}= & Q_{\mathrm{SL}}-Q_{\mathrm{SL} 0} \\
= & \frac{V_{\mathrm{ES}} V_{\mathrm{NC}} \sin \left(\theta_{\mathrm{ES}}\right)}{Z_{\mathrm{NC}}}+\frac{V_{\mathrm{NC}}^{2} \sin \left(\phi_{\mathrm{NC}}\right)}{Z_{\mathrm{NC}}} \\
& -\frac{V_{C}^{2} \sin \left(\phi_{\mathrm{NC}}\right)}{Z_{\mathrm{NC}}} .
\end{aligned}
$$

The capability curves for an SLBC in Fig. 5(a) and (b) show that available range of $\Delta P_{\mathrm{SL}}$ (horizontal intercepts) is larger compared to SLES for identical converter capacity. The available range of $\Delta Q_{\mathrm{SL}}$ (vertical intercepts) remains similar

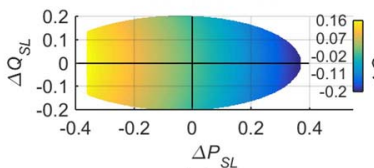

(a)

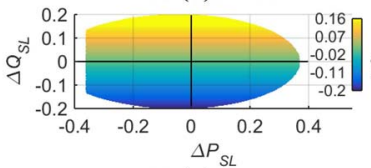

(c)

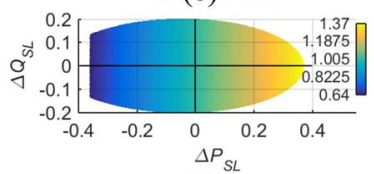

(e)

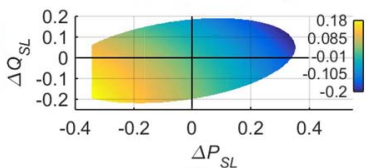

(b)

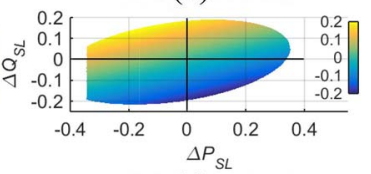

(d)

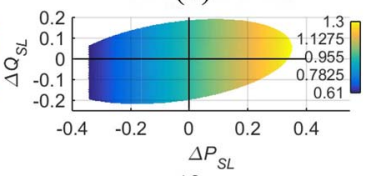

(f)
Fig. 5. Capability of SLBC. (a) and (b) Active compensation required. (c) and (d) Reactive compensation required. (e) and (f) Active power consumption of $\mathrm{NC}$ load corresponding to the change in active power ( $x$-axis) and reactive power ( $y$-axis) of an SLBC for two different pfs.

to that of an SLES. It can be seen that for 0.95 lagging pf load, the available range in the first and the third quadrant is larger with an SLBC as compared to an SLES which allows more flexibility in voltage control. However, the available range in the second and fourth quadrant is less for an SLBC than an SLES which could be a limitation in the case of simultaneous voltage and primary frequency control.

As in the case of an SLES, the $P-Q$ capability of an SLBC is limited for negative $\Delta P_{\mathrm{SL}}$. The active $\left(P_{\mathrm{ES}}\right)$ and reactive $\left(Q_{\mathrm{ES}}\right)$ powers required by converter 1 (ES) for operation in different quadrants (for both pfs) are shown in Fig. 5(a)-(d). The rating of converter 2 depends $P_{\mathrm{ES}}$. It can be seen that, unlike an SLES, $\Delta P_{\mathrm{NC}}$ is very similar to $\Delta P_{\mathrm{SL}}$ for both pfs [Fig. 5(e) and (f)].

The capability curves in this section are valid for a constant impedance type NC load. Similar curves for a constant current type (or other forms of voltage-dependant) NC load are not included here due to space restriction. The available range for a constant current type load would be smaller due to the linear power voltage relationship. An SLBC will still be effective for constant current type NC loads while an SLES could fail due to the opposite signs of $P_{\mathrm{ES}}$ and $\Delta P_{\mathrm{NC}}$, and the linear power voltage relationship.

\section{Control of SLBC}

The control objective is to vary the active and reactive power consumption of the SLBC within its capability in order to regulate the supply voltage. The control loop is shown in Fig. 6.

The voltage error $(\Delta V)$, which is the difference between the reference voltage $\left(V_{\text {ref }}\right)$ and the measured supply voltage $\left(V_{C}\right)$, is fed to a proportional-integral (PI) controller through a dead-band. The output of the PI controller is weighted according to the equivalent $R / X$ ratio of the network to derive the required change in active $\left(\Delta P_{\mathrm{SL}}\right)$ and reactive $\left(\Delta Q_{\mathrm{SL}}\right)$ power consumption of the SL. The SL model uses $\Delta P_{\mathrm{SL}}$, $\Delta Q_{\mathrm{SL}}$, and the measured supply voltage $V_{C}$, to calculate 


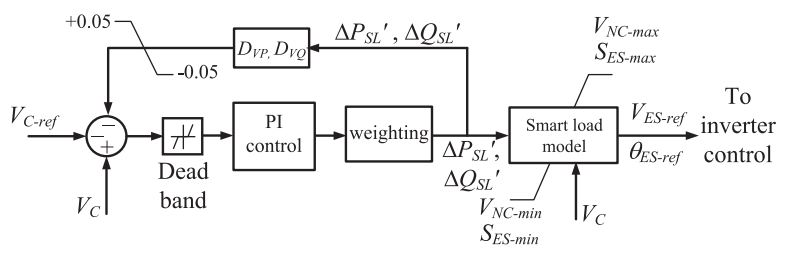

Fig. 6. Control loop for voltage control using an SLBC.

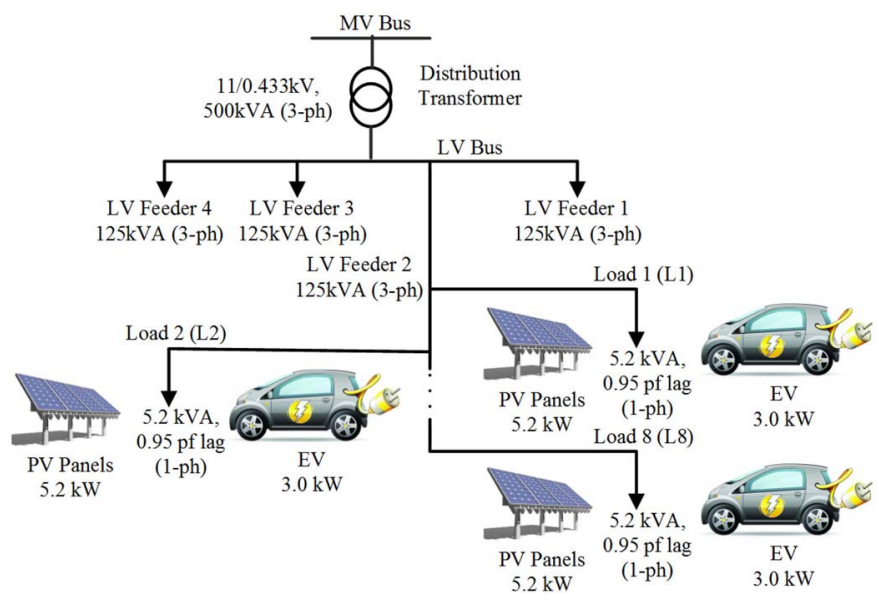

Fig. 7. Segment of an LV distribution network [14] with PV generation and EV charging points.

the reference voltage magnitude $\left(V_{\mathrm{ES}-\text {-ref }}\right)$ and phase angle $\left(\theta_{\mathrm{ES}-\mathrm{ref}}\right)$ for converter 2 . Appropriate limits on allowable variation in $\mathrm{NC}$ load voltage $\left(V_{\mathrm{NC}-\mathrm{min}}\right.$ and $\left.V_{\mathrm{NC}-\mathrm{max}}\right)$ and the apparent power $\left(S_{\mathrm{ES}-\mathrm{min}}\right.$ and $S_{\mathrm{ES} \text {-max }}$ ) of the compensator are imposed within the SL model. Droop gains ( $D_{\mathrm{VP}}$ and $\left.D_{\mathrm{VQ}}\right)$ are used to update the voltage reference $\left(V_{\text {ref }}\right)$ within the allowed limits \pm 0.05 p.u. to ensure that the SLs connected at different buses do not work against each other [20].

\section{CASE STUdy}

\section{A. Study System}

A section of a typical U.K. distribution system [14] as shown in Fig. 7 is chosen for this paper. Only the LV side is modeled in detail while the MV bus is considered to be tightly regulated at 1.0 p.u. The system consists of a $500 \mathrm{kVA}$ distribution transformer and a radial LV distribution system with four identical feeders. The transformer taps are set at $-5 \%$ to ensure that the voltage regulation at the far end is less than $5 \%$ at full load. One LV feeder is modeled in detail while the rest are represented as equivalent loads. The 300-m-long LV feeder has two cable sections each $150 \mathrm{~m}$ long with impedances $0.164+j .074$ and $0.32+j .075 \Omega / \mathrm{km}$.

There are eight equally spaced single-phase loads connected to each phase of the LV feeder. Each load has a peak power rating of $5.2 \mathrm{kVA}$ and a pf of 0.95 lagging. Each load is connected to the main LV feeder through a $30-\mathrm{m}(0.215+j .0125 \Omega / \mathrm{km})$ service cable. For the base case of this paper, the load at each bus is equally divided into normal and SLs. A typical U.K. domestic daily load profile as shown in Fig. 8 [21] is considered.

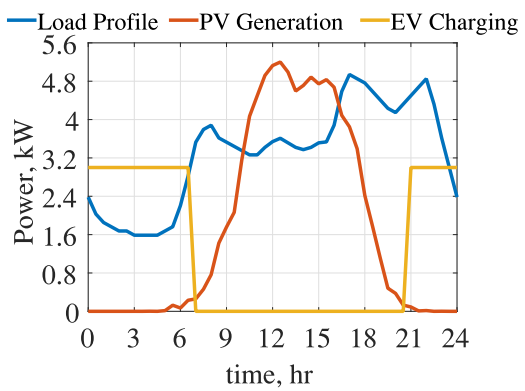

Fig. 8. Half-hourly variation in load, PV output, and EV charging power.

To simulate voltage disturbances, a PV panel with a peak power of $5.2 \mathrm{~kW}$ and an EV charging facility of $3.0 \mathrm{~kW}$ are included at each load terminal. A typical PV output profile is generated using a half-hourly average solar irradiation data. The EV charging power is assumed to be constant as [22] suggests that the EVs will require a constant power during the charging cycle. The half-hourly variation in load, PV generation, and EV charging profile are shown in Fig. 8. Over-voltage occurs during the day time when the PV generation is close to its peak value while EV charging near peak load causes under-voltage.

For the purpose of this paper, an ideal controller with zero response time was considered for all SLs. Also, the converters were assumed to be ideal. For a practical SL, it is necessary to consider the nonideal behavior of the phase lock loop (PLL), the response time of the converter control, and the dynamics of the dc link, which might cause the phase angle to change slightly from the reference angle in transient state to account for the losses in the converter. As only steady-state values are presented in the simulation results, the controllers, the PLLs, and the converters were considered to be ideal.

\section{B. Over- and Under-Voltage Conditions}

Simulations have been carried out in MATLAB/Simulink to compare the effectiveness of SLQs and SLBCs in voltage control under various conditions. Results for the load L8 connected at the far end of the feeder are shown in Figs. 9 and 10 .

Fig. 9(a) shows that with a normal load (NL) (red trace), the supply voltage at $L 8$ increases up to 1.086 p.u. around the midday while it reduces to 0.925 p.u. during the late evening peak. The SLBC (black trace) is able to restore the voltage back within the permissible range of \pm 0.05 p.u. The SLQ (green trace) is able to restore the voltage in the case of under-voltage, but it cannot do so during the over-voltage situation. For supply voltage within the allowed range of \pm 0.05 p.u., the SLQ and the SLBC behave like an NL which results in overlapping traces. As expected, a larger variation in the voltage across $\mathrm{NC}$ load $\left(V_{\mathrm{NC}}\right)$ is observed for the SLBC as compared to that for the SLQ [Fig. 9(b)]. The corresponding compensator (ES) voltage and its phase angle are shown in Fig. 9(c) and (d).

The voltage responses shown above can be explained by observing the power variations as shown in Fig. 10. During the over-voltage (under-voltage) condition, both active and reactive powers of the SLBC increase above (decrease below) the 


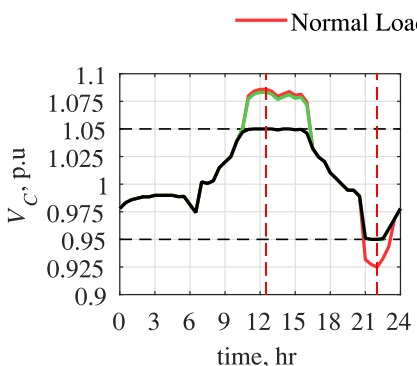

(a)

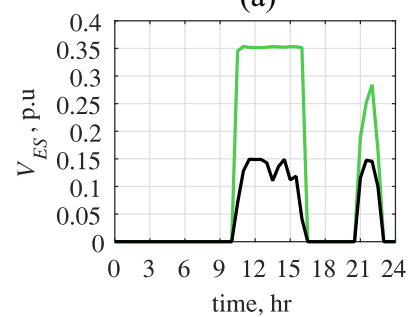

(c)

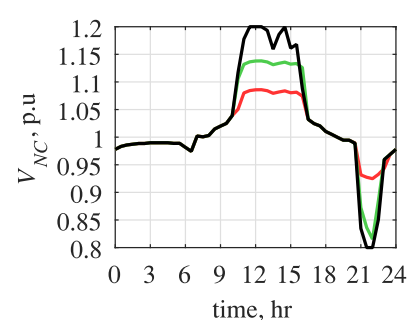

(b)

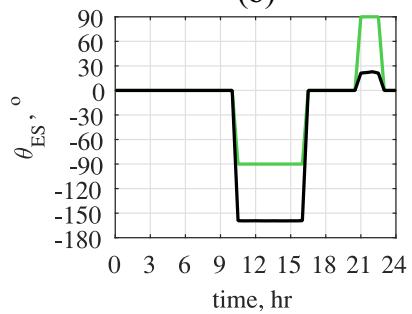

(d)
Fig. 9. Variation in (a) supply voltage at $L 8$, (b) voltage across NC load, (c) compensator voltage magnitude, and (d) phase angle over $24 \mathrm{~h}$.

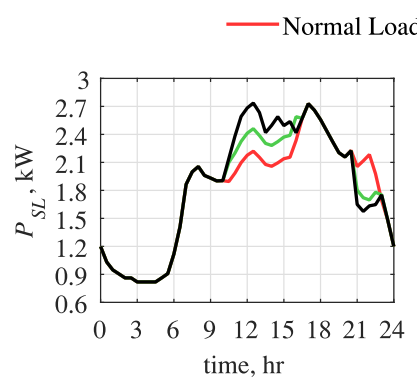

(a)

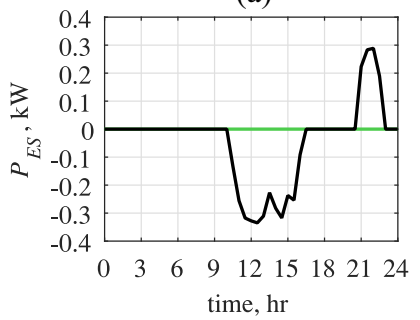

(c)

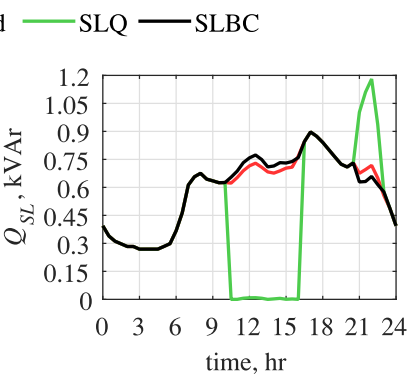

(b)

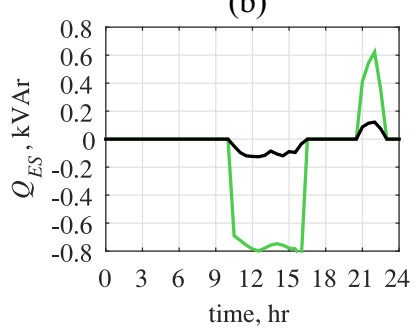

(d)
Fig. 10. Variation in (a) active and (b) reactive power of SL, and (c) active and (d) reactive power of the compensator over $24 \mathrm{~h}$.

normal value to restore the system voltage. However, for the SLQ, an increase in active power is accompanied with a large decrease in the reactive power which makes it less effective in regulating the supply voltage. A similar conflicting trend is observed during the under-voltage situation. Nonetheless, the voltages can be restored to 0.95 p.u. as the under-voltage event in this paper is not as severe as the over-voltage one as explained in the following paragraphs.

In the simulation study, the SLs are shown to mitigate sustained voltage problems over reasonably long-time frames (hours). In practice, several NC loads would not be able to tolerate sustained voltage deviations. Hence, it is likely that the such SLs would regulate the voltage only in the short-term

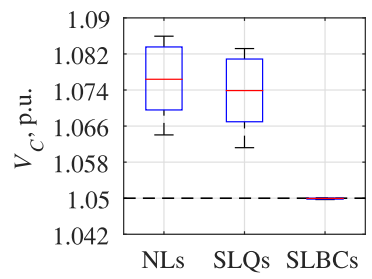

(a)

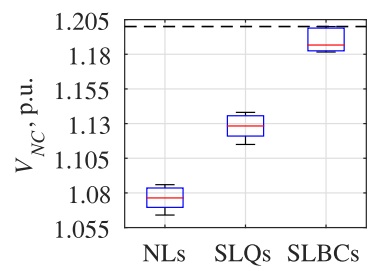

(c)

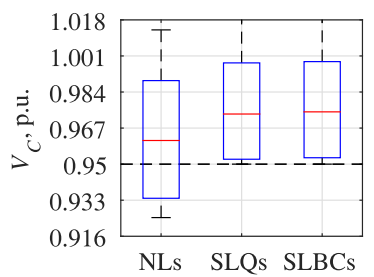

(b)

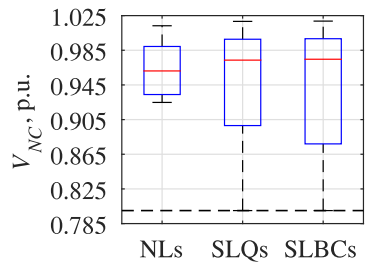

(d)
Fig. 11. Box plots for voltage across (a) and (b) supply/mains and (c) and (d) NC loads for under- and over-voltage events with NLs, SLQs, and SLBCs. On each box, the central mark is the median, the edges of the box are the 25th and 75th percentiles, and the whiskers extend to the most extreme voltage values.

until other voltage control measures such as transformer tap changer and network reconfiguration are activated.

To complement the transient responses at a particular bus (L8), the collective response of all the SLs is captured through boxplots for voltages at all load buses as shown in Fig. 11 for the worst over- and under-voltage conditions marked (by red-dotted lines) in Fig. 9(a). For the over-voltage case [Fig. 11(a)], all NL voltages are above 1.05 p.u. with a few node voltages as high as 1.085 p.u. With the SLQs activated, the node voltages are still above 1.05 p.u. although some of the node voltages have been improved (reduced). Unlike SLQs, SLBCs are able to maintain the voltages within 1.05 p.u. at all the nodes. In fact, all the node voltages are held close to 1.05 p.u. (narrow boxplot) because of the droop setting. This improved voltage regulation is achieved through a wider variation in voltage (and hence power) across the NC loads [as shown in Fig. 11(b)] which is limited to \pm 0.2 p.u.

Fig. 11(b) shows the results for the under-voltage case where only some of the node (at the far end of the feeder) voltages drift below 0.95 p.u. while the voltages at some nodes (at the start of the feeder) are still above 1.0 p.u. The reference voltages for the SLs at different nodes are adjusted by their respective droop settings. It is evident that the under-voltage event is less severe than the over-voltage event as only a few nodes violate the under-voltage limit. Both SLQs and SLBCs are able to restore all voltages within the allowed range. In both cases, the NC load voltages are reduced right down to their lower limit of 0.8 p.u. as shown in Fig. 11(d).

The total apparent power capacity of the converters is expressed as a percentage of the total SL apparent power (kVA) rating and is shown separately for the SLQs and the two converters of SLBCs in Fig. 12. The total capacity required for both the converters of SLBCs is smaller than that of SLQs. This paper, therefore, shows that SLBCs can produce better voltage regulation than SLQs especially, during over-voltage conditions. 


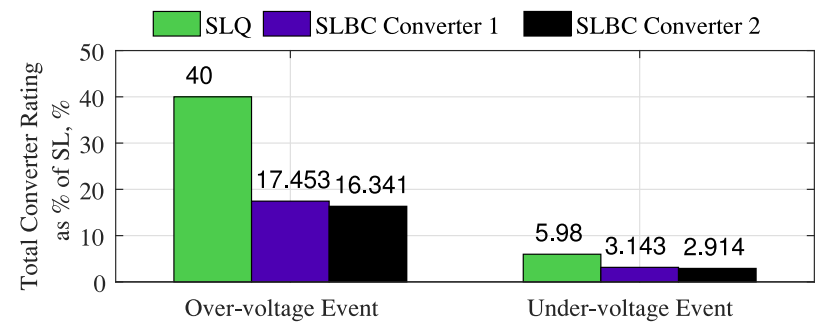

Fig. 12. Total reactive capacity of the converters for SLQ and SLBC expressed as a percentage of the SL rating.

In terms of costs, the two relatively lower rated converters for an SLBC will cost less than the converter required for an SLQ. For both, power losses will be incurred under normal condition due to the load current flowing through the series converter even when there is no compensation. This is similar to the power losses incurred in any power electronic interface (e.g., drive circuit) for loads unless special arrangements are made to bypass the series converter under normal condition through the use of hybrid (mechanical-electronic) switching. The efficiency of both SLQ and SLBC will be comparable under normal conditions when the parallel converter of the SLBC is not active. During compensation, an additional power loss will be incurred in the parallel converter of the SLBC, which is operated at unity pf and is only rated at the maximum value of the active power exchanged by the series converter. This additional power loss should be taken into account while comparing the efficiency of an SLQ and an SLBC.

\section{Sensitivity Analysis}

The ability of the SLs (both SLQs and SLBCs) to regulate the supply voltage depends on several factors including the pf of the NC load, proportion of $\mathrm{NC}$ load in the system (larger proportion will result in better voltage control), and $R / X$ ratio of the network. These factors could vary from one system to other which calls for a sensitivity analysis. For the sensitivity studies presented in this section, the voltage variation across the NC load is limited to $20 \%$. The apparent power capacity of each converter in an SLBC is set to $20 \%$ of the corresponding NC load rating (as shown in Section II-C3). For the SLQs, the converter rating is chosen to be $40 \%$ of the respective NC load rating which corresponds to the maximum positive value of $\Delta P_{\mathrm{SL}}$ as discussed in Section II-C1.

To study the impact of varying the above parameters on voltage regulation, an aggregated voltage regulation index (VRI) [12] is defined as follows:

$$
\operatorname{VRI}(\text { in } \%)=\frac{\sum_{i=1}^{N_{\text {bus }}}\left\{\max _{t}\left|V_{i}(t)-V_{\text {ref }}\right| \times W_{\mathrm{vi}}\right\}}{N_{\text {bus }}} \times 100
$$

where

$$
\begin{aligned}
W_{\mathrm{vi}} & =1 \text { if } \max _{t}\left|V_{i}(t)-V_{\text {ref }}\right| \leq 0.05 \text { p.u. } \\
& =2 \text { if } 0.05 \text { p.u. }<\max _{t}\left|V_{i}(t)-V_{\text {ref }}\right| \leq 0.1 \text { p.u. } \\
& =10 \text { if } \max _{t}\left|V_{i}(t)-V_{\text {ref }}\right|>0.1 \text { p.u. }
\end{aligned}
$$

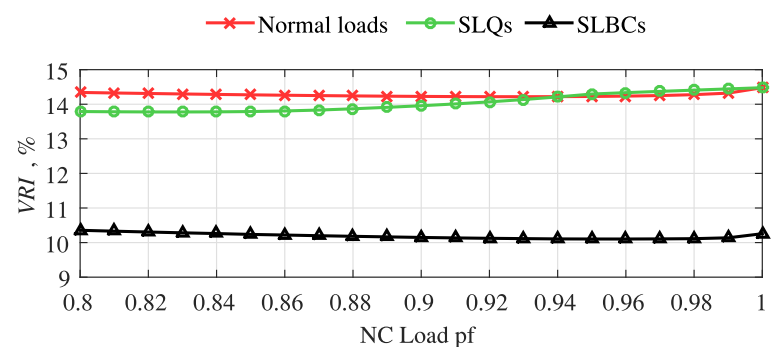

(a)

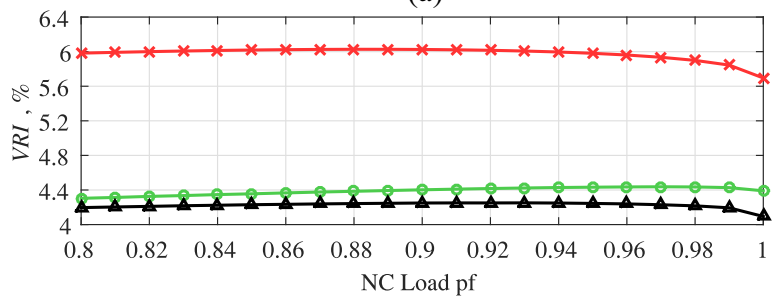

(b)

Fig. 13. Impact of NC load pf on VRI (27) for (a) over- and (b) under-voltage events.

In the above expression, $V_{i}(t)$ is the p.u. value of voltage at the $i$ th bus as a function of time, $V_{\text {ref }}$ is the reference voltage in p.u., $N_{\text {bus }}$ is the total number of buses, and $W_{\text {vi }}$ is a weighted penalty factor to impose extra penalty if the voltage variation is outside the allowed range. The maximum deviation from reference value over time is considered for each bus.

The effect of variation in NC load pf on VRI is shown in Fig. 13. For low pfs (0.8), the SLQs are not able to improve VRI significantly compared to the NLs for an overvoltage event [Fig. 13(a)]. This is due to a large negative value of $\Delta Q_{\mathrm{SL}}$ associated with any positive value of $\Delta P_{\mathrm{SL}}$ [Fig. 3(a)]. While there is an increase in active power, the large decrease in reactive power tends to increase the voltage. As the pf approaches unity, the maximum positive value of $\Delta P_{\mathrm{SL}}$ approaches zero. Under these conditions, the VRI with SLQs approaches that for NLs (i.e., no control). The SLQs achieve better voltage regulation for under-voltage event [Fig. 13(b)]. This is due to a wider operating range available for negative values of $\Delta P_{\mathrm{SL}}$ and relatively smaller associated positive values of $\Delta Q_{\mathrm{SL}}$, especially, for lower pfs.

The SLBCs are able to achieve better voltage regulation (i.e., lower VRI) than the SLQs over a range of pfs for both over- and under-voltage events. This is due to wider available operating range especially, for positive $\Delta P_{\mathrm{SL}}$ as explained in Section II-C3.

The equivalent $R / X$ ratio of the network plays an important role in voltage control using SLs. It not only determines the severity of the voltage problem with NLs but also influences the effectiveness of the SLQs and SLBCs. The impact of the system $R / X$ ratio on VRI is shown in Fig. 14. The VRI for NLs (no control) increases for a higher $R / X$ ratio as both PVs and EVs exchange only active power with the network.

From Fig. 14, it is evident that the SLQs in reactive power $(Q)$ control are more effective in controlling the voltage for a lower $R / X$ ratio while the SLQs in active power $(P)$ control are more effective for a higher $R / X$ ratio. The SLQs cannot produce acceptable voltage regulation for any $R / X$ ratio 


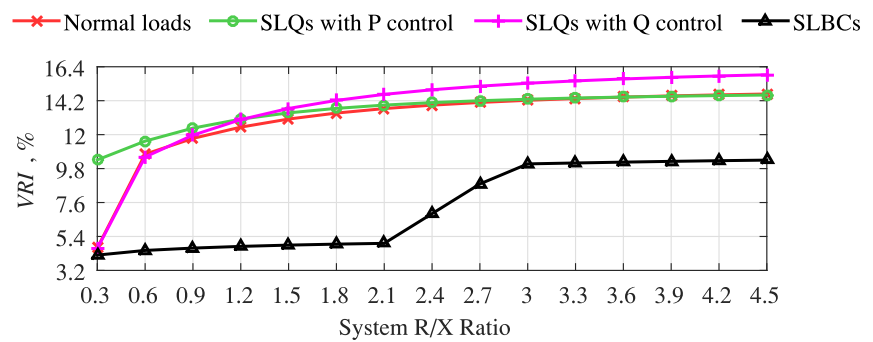

(a)

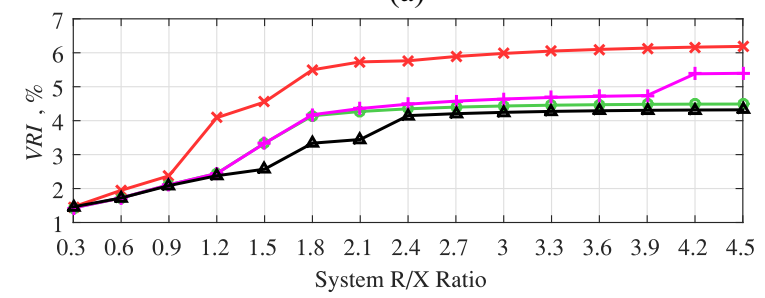

(b)

Fig. 14. Impact of system $R / X$ ratio on VRI (27) for (a) over- and (b) undervoltage events.

in the case of an over-voltage event. On the other hand, the SLBCs are able to maintain a low VRI for a wider range of $R / X$ ratio. The controller can be tuned to determine the optimal performance based on the system $R / X$ ratio as the active and the reactive power consumption of an SLBC can be controlled independently. For large values of $R / X$ (i.e., $R / X>3$ ), VRI increases sharply due to the converter capacity (20\%) limitations. Nonetheless, VRI with the SLBCs is still lower than that with SLQs. For an under-voltage event, both SLQs and SLBCs produce acceptable voltage regulation for a range of $R / X$ ratio.

\section{CONCLUSION}

SLBCs can be used to effectively control the voltage in an LV network. SLBCs perform better compared to SLQs especially, in the case of over-voltage events caused by PV generation. While the performance of SLQs depend on the $R / X$ ratio of the network, SLBCs can ensure acceptable voltage regulation over a wider range of $R / X$ ratios. Moreover, SLBCs can achieve better voltage regulation with less total converter power capacity than SLQs. This improvement in voltage regulation with SLBCs would be more pronounced in MV networks where the $R / X$ ratio is relatively less. In this paper, the SLs are shown to mitigate sustained voltage problems over reasonably long-time frames (hours). In practice, depending on the nature of the SLs it is likely that these would regulate the voltage only in the shortterm (minutes) until other voltage control measures such as transformer tap changer and network reconfiguration are activated.

An SLBC would require one additional power converter compared to an SLQ. For both, the series converter incurs power loss due to the load current under normal operation (i.e., when the compnesator/ES is not active) while the parallel converter in an SLBC is virtually out of operation when the compensator is not active. Therefore, despite requiring two converters, an SLBC has similar efficiency as an SLQ under normal operation. From a cost and compactness point of view, SLBCs are likely to be more suitable for high power loads which are mostly connected at the MV level. Nonetheless, this paper on the LV network demonstrates the technical potential of the SLBCs in mitigating voltage problems in distribution networks.

Supporting data is available on request: please contact cap-publications@imperial.ac.uk.

\section{REFERENCES}

[1] M. H. J. Bollen, Understanding Power Quality Problems: Voltage Sags and Interruptions. Piscataway, NJ, USA: IEEE Press, 2000.

[2] Y. Li and P. A. Crossley, "Monte Carlo study on impact of electric vehicles and heat pumps on LV feeder voltages," in Proc. 12th IET Int. Conf. Develop. Power Syst. Protect. (DPSP), Copenhagen, Denmark, 2014, pp. 1-6.

[3] P. M. S. Carvalho, P. F. Correia, and L. A. F. Ferreira, "Distributed reactive power generation control for voltage rise mitigation in distribution networks," IEEE Trans. Power Syst., vol. 23, no. 2, pp. 766-772, May 2008.

[4] K. Christakou, D.-C. Tomozei, J.-Y. Le Boudec, and M. Paolone, "GECN: Primary voltage control for active distribution networks via real-time demand-response," IEEE Trans. Smart Grid, vol. 5, no. 2, pp. 622-631, Mar. 2014.

[5] A. R. Di Fazio, G. Fusco, and M. Russo, "Decentralized control of distributed generation for voltage profile optimization in smart feeders," IEEE Trans. Smart Grid, vol. 4, no. 3, pp. 1586-1596, Sep. 2013.

[6] F. Marra et al., "Improvement of local voltage in feeders with photovoltaic using electric vehicles," IEEE Trans. Power Syst., vol. 28, no. 3, pp. 3515-3516, Aug. 2013.

[7] M. N. Kabir, Y. Mishra, G. Ledwich, Z. Y. Dong, and K. P. Wong, "Coordinated control of grid-connected photovoltaic reactive power and battery energy storage systems to improve the voltage profile of a residential distribution feeder," IEEE Trans. Ind. Informat., vol. 10, no. 2, pp. 967-977, May 2014.

[8] K. Christakou, D.-C. Tomozei, M. Bahramipanah, J.-Y. Le Boudec, and M. Paolone, "Primary voltage control in active distribution networks via broadcast signals: The case of distributed storage," IEEE Trans. Smart Grid, vol. 5, no. 5, pp. 2314-2325, Sep. 2014.

[9] P. N. Vovos, A. E. Kiprakis, A. R. Wallace, and G. P. Harrison, "Centralized and distributed voltage control: Impact on distributed generation penetration," IEEE Trans. Power Syst., vol. 22, no. 1, pp. 476-483, Feb. 2007.

[10] S. Y. Hui, C. K. Lee, and F. F. Wu, "Electric springs-A new smart grid technology," IEEE Trans. Smart Grid, vol. 3, no. 3, pp. 1552-1561, Sep. 2012.

[11] X. Luo et al., "Distributed voltage control with electric springs: Comparison with STATCOM," IEEE Trans. Smart Grid, vol. 6, no. 1, pp. 209-219, Jan. 2015.

[12] Z. Akhtar, B. Chaudhuri, and S. Y. R. Hui, "Primary frequency control contribution from smart load with reactive compensation," IEEE Trans. Smart Grid, vol. 6, no. 5, pp. 2356-2365, Sep. 2015.

[13] S. Y. R. Hui, C. K. Lee, and F. F. Wu, "Power control circuit and method for stabilizing a power supply," U.S. Patent U.S. 0080 420, Apr. 5, 2012.

[14] S. Ingram, S. Probert, and K. Jackson, "The impact of small scale embedded generation on the operating parameters of distribution networks," Dept. Trade Ind., London, U.K., Tech. Rep. K/EL/00303/04/01, 2003.

[15] C. K. Lee, K. L. Cheng, and W. M. Ng, "Load characterisation of electric spring," in Proc. IEEE Energy Convers. Congr. Expo. (ECCE), Denver, CO, USA, 2013, pp. 4665-4670.

[16] S. Wong and S. Pelland, "Demand response potential of water heaters to mitigate minimum generation conditions," in Proc. IEEE Power Energy Soc. Gen. Meeting (PES), Vancouver, BC, Canada, 2013, pp. 1-5.

[17] C. K. Lee, S. Li, and S. Y. Hui, "A design methodology for smart LED lighting systems powered by weakly regulated renewable power grids," IEEE Trans. Smart Grid, vol. 2, no. 3, pp. 548-554, Sep. 2011. 
[18] L. Ning, X. Yulong, H. Zhenyu, F. Puyleart, and S. Yang, "Load component database of household appliances and small office equipment," in Proc. IEEE Power Energy Soc. Gen. Meeting, Pittsburgh, PA, USA, 2008, pp. 1-5.

[19] H. Fujita and H. Akagi, "The unified power quality conditioner: The integration of series and shunt-active filters," IEEE Trans. Power Electron. vol. 13, no. 2, pp. 315-322, Mar. 1998.

[20] C. K. Lee, N. R. Chaudhuri, B. Chaudhuri, and S. Y. R. Hui, "Droop control of distributed electric springs for stabilizing future power grid," IEEE Trans. Smart Grid, vol. 4, no. 3, pp. 1558-1566, Sep. 2013.

[21] (Jan. 16, 2015). UKERC-EDC Data Archive. [Online]. Available: http://data.ukedc.rl.ac.uk/browse/edc/Electricity/LoadProfile/data

[22] Q. Kejun, Z. Chengke, M. Allan, and Y. Yue, "Modeling of load demand due to EV battery charging in distribution systems," IEEE Trans. Power Syst., vol. 26, no. 2, pp. 802-810, May 2011.

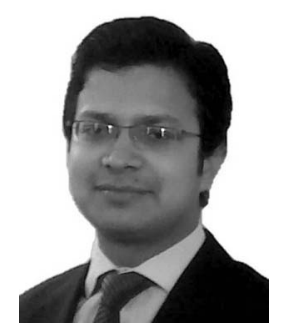

Zohaib Akhtar (S'14) received the B.Sc. (Hons.) and M.Sc. degrees in electrical engineering from the University of Engineering and Technology, Lahore, Pakistan, in 2008 and 2011, respectively. He is currently pursuing the Ph.D. degree with Imperial College London, London, U.K.

His current research interests include renewable energy, power distribution, and smart grids.

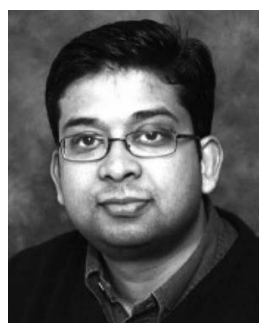

Balarko Chaudhuri (M'06-SM'06) received the $\mathrm{Ph} . \mathrm{D}$. degree in electrical and electronic engineering from Imperial College London, London, U.K., in 2005 .

He is currently a Senior Lecturer with the Control and Power Research Group, Imperial College London. His current research interests include electric power transmission systems, control theory, smart grids, and renewable energy.

Dr. Chaudhuri is an Associate Editor of the IEEE SYSTEMS JOURNAL and Control Engineering Practice. $\mathrm{He}$ is a member of the Institution of Engineering and Technology (IET) and CIGRE.

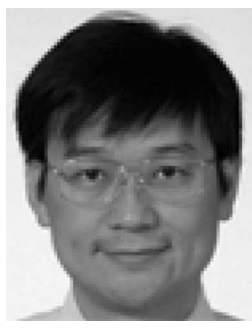

Shu Yuen Ron Hui (M'87-SM'94-F'03) received the B.Sc. (Hons.) degree in electrical and electronic engineering from the University of Birmingham, Birmingham, U.K., in 1984, and the D.I.C. and $\mathrm{Ph} . \mathrm{D}$. degree in electrical engineering from Imperial College London, London, U.K., in 1987.

He currently holds the Philip Wong Wilson Wong Chair Professorship with the University of Hong Kong, Hong Kong. Since 2010, he has concurrently held a part-time Chair Professorship in Power Electronics with Imperial College London. He has published over 300 technical papers, including about 200 refereed journal publications and book chapters. Over 55 of his patents have been adopted by industry.

Prof. Hui was a recipient of the IEEE Rudolf Chope Research and Development Award from the IEEE Industrial Electronics Society, the IET Achievement Medal (Crompton Medal) from the Institution of Engineering and Technology in 2010, and the 2015 IEEE William E. Newell Power Electronics Award. He is a Fellow of the Australian Academy of Technological Sciences and Engineering. $\mathrm{He}$ is an Associate Editor of the IEEE TRANSACTIONS ON POWER ElECTRONICS and the IEEE TRANSACTIONS ON INDUSTRIAL ELECTRONICS. He is an Editor of the IEEE JOURNAL OF EMERGING AND SELECTED TOPICS OF POWER EleCtronics. 